Hoekstra, J. \& L. Marácz. 1989a. 'Some implications of I-to-C-movement in Frisian'. In: H. Bennis \& A. van Kemenade (eds.), Linguistics in the Netherlands 1989. Foris Publications, Dordrecht, 81-90.

Hoekstra, J. \& L. Marácz. 1989b. "The Position of Inflection in WestGermanic'. Working Papers in Scandinavian Syntax 44:75-88.

Karsten, G. 1931. Het dialect van Drechterland. Muusses, Purmerend.

Laan, K. ter. 1952². Nieuw Groninger Woordenboek. Noordhoff, Groningen-Djakarta.

Lorenzen, J. 1981. Halligfriesisches Lesebuch / Halifreesk Leeseböök. Nordfriisk Instituut, Bräist.

Meer, G. van der. 1988. 'Reported Speech and the Position of the Finite Verb'. Leuvense Bijaragen 77:301-24.

. Milsark, G.L. 1988. 'Singl-ing'. Linguistic Inquiry 19:611-34.

Platzack, C. \& A. Holmberg. 1989. 'The role of AGR and finiteness in Germanic VO-languages'. Working Papers in Scandinavian Syn$\operatorname{tax} 43: 51-76$

Riemsdijk, H. van. 1982. 'A Note on Case Absorption'. Wiener Linguistische Gazette 28-29:72-83.

Schmidt-Petersen, J.\& J. Craigie. 1928. The North Frisian Dialect of Föhr and Amrum. Hutchen, Edinburgh.

Schuurman, I. 1987. 'Incorporation in the Groningen dialect'. In: F. Beukema \& P. Coopmans (eds.), Linguistics in the Netherlands 1987. Foris Publications, Dordrecht, 185-94.

Schuurman, I. \& A. Wierenga. 1986. 'Syntactische nomen-incorporatie bij infinitieven en deelwoorden'. TABU 16:339-50.

Schuurman, I. \& A. Wierenga. 1990. 'Het Gronings: 'Verb-raising' in soorten en maten'. In: G. de Schutter et al. (eds.), Dialectsyntaxis (Taal en Tongval, themanummer 3), 74-86.

Wilts, O. 1980. 'Zu einigen Neufestsetzungen der föhring-amringer Orthographie'. Nordfriesisches Jahrbuch 16:141-45.

Wurdenbuk = Wurdenbuk för Feer an Oomram, mit Hilfe von Elene Braren und Nickels Hinrichsen zusammengestellt von Ommo Wilts. Verlag Jens Quedens, Insel Amrum (1986).

\section{Siebren Dyk}

WARUM GIBT ES IM WESTERLAUWERSSCHEN UND FÖHRER FRIESISCHEN EINE NOMENINKORPORATION?

V. U.F. Falting, A. G. H. Waller 8 o. hilt (eas.) : studien I.

Acener Univenits Press.

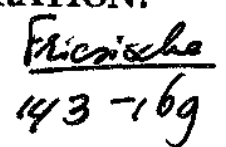

In Dyk (1992) habe ich die These aufgestellt, daß im Westfriesischen, d.h. im Friesischen des im Norden der Niederlande gelegenen westerlauwersschen Friesland, das Phänomen der Nomeninkorporation auftritt. In diesem Artikel ${ }^{*}$ möchte ich untersuchen, ob in dem auf der Insel Föhr gesprochenen Friesisch etwas Ähnliches festzustellen ist (Abschnitt 2). Wie sich zeigen wird, ist das tatsächlich der Fall. Im Abschnitt 3 möchte ich die Frage beantworten, warum es im Friesischen überhaupt eine Nomeninkorporation gibt. Einfachheitshalber werden die beiden Varianten des Friesischen entsprechend ihrer einheimischen Bezeichnung als 'Frysk' und 'Fering' gekennzeichnet.

\section{Einleitung}

Bevor wir auf die Nomeninkorporation im Fering eingehen, möchten wir darlegen, was dieses Phänomen überhaupt beinhaltet. Ich greife hierbei auf meine Grundschulerfahrungen im Grammatikunterricht zurück. In meiner Jugend galt als Standardbeispiel eines transitiven Satzes:

(1) De slager slacht de koe

('Der Schlachter schlachtet die Kuh')

Der erste Satz ist niederländisch, denn wir hatten damals kein Friesisch auf der Grundschule, doch ist das Prinzip, um das es 


\section{Siebren Dyk}

geht, in beiden Sprachen und auch im Hochdeutschen dasselbe. Wir haben in diesem Satz drei Konstituenten: ein Subjekt (de slager), ein Objekt (de koe) und ein Verb (slacht). Man kann dabei behaupten, daß das Verb mit dem Objekt etwas enger verknüpft ist und darum eine höhere Konstituente bildet. Rein graphisch, also ohne damit weitere theoretische Konsequenzen zu verknüpfen, läßt sich dieser Satz wie folgt darstellen:

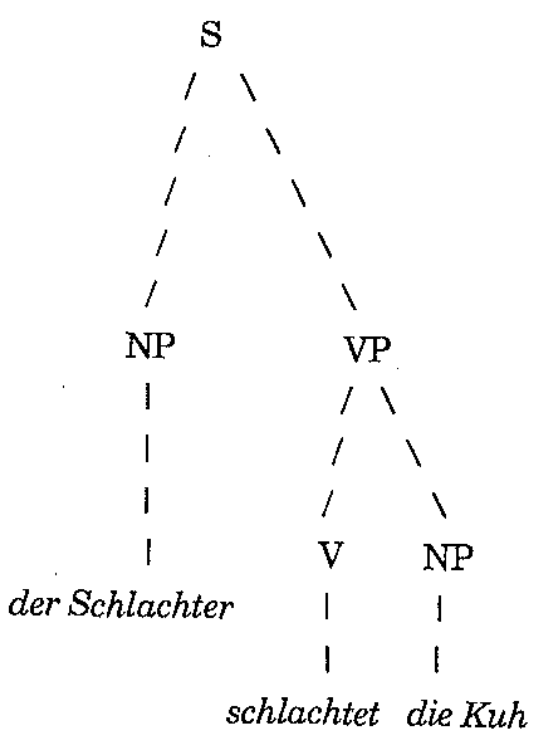

Dies alles ist selbstverständlich nichts Neues und gilt für alle drei obengenannten Sprachen, also für das Niederländische, das Frysk und das Hochdeutsche. Alle drei Sprachen zeigen auch die gleiche Reaktion, wenn man den Satz innerhalb einer untergeordneten Nebensatzkonstruktion gebraucht, in der das Verb am Satzende steht:

(3) (Ich höre) daß der Schlachter die Kuh schlachtet

\section{Nomeninkorporation}

(Ik hoor) dat de slager de koe slacht (Ik hear) dat de slachter de ko slachtet

Im Frysk ist es allerdings auch möglich, den Artikel wegzulassen, was für die beiden anderen Sprachen nicht gilt:

(4) a. *.. daß der Schlachter Kuh schlachtet

b. *... dat de slager koe slacht

c. ... dat de slachter ko slachtet

$\mathrm{Da}$ das Objekt in (4c) mit dem Verb ein Wort bildet, ist es korrekter, wie bei Komposita üblich, beide Wörter zusammenzuschreiben:

(5) ... dat de slachter koslachtet

Daß wir es nur mit einem Wort zu tun haben, wird auch dadurch ersichtlich, daß in Hauptsätzen das Objekt ebenfalls vor dem Verb steht:

(6) De slachter koslachtet

Dies steht im Gegensatz zu den syntaktischen Konstruktionen mit einem selbständigen Objekt, wie die fryske Variante von (1) zeigt:

(7) De slachter slachtet de ko

Man kann also sagen, daß Objekt und Verb hier ein komplexes Verb bilden, das als Ganzes den zweiten Platz im Satz besetzt (der sogenannte 'Verb Second'-Effekt). Graphisch ist (6) wiederzugeben als 


\section{Siebren Dyk}

(8)

\begin{tabular}{|c|c|}
\hline \multicolumn{2}{|c|}{$\mathrm{S}$} \\
\hline 1 & 1 \\
\hline 1 & 1 \\
\hline I & 1 \\
\hline 1 & 1 \\
\hline $\mathrm{NP}$ & VP \\
\hline 1 & 1 \\
\hline 1 & $\mathrm{~V}$ \\
\hline 1 & 1 \\
\hline e slachter & koslachte \\
\hline
\end{tabular}

Das Objekt scheint hier ein Teil des Verbs geworden zu sein, daher die Bezeichnung 'Nomeninkorporation'.

Die Nomeninkorporation ist insbesondere bekannt aus 'exotischen' Sprachen, vor allem aus Indianersprachen. Hierzu ein Beispiel aus dem Onondaga, einer Sprache im Nordosten der Vereinigten Staaten:

\section{(9) a. wa hahninú ne? oyEkwa? TNS.3sg.3N.buy.ASP ART 3N.tobacco.NM 'He bought the tobacco' \\ b. wa hayEkahnínu? \\ TNS.3sg.3N.tobacco.buy.ASP \\ 'He bought tobacco' （aus Rosen 1989:295)}

Das Phänomen der Nomeninkorporation ist in den letzten Jahren von theoretischen Sprachwissenschaftlern im Rahmen einer Diskussion über den Platz der Morphologie im Sprachmodell ausgiebig untersucht worden. Vor allem Baker (1988) stellt eine syntaktische Ableitung dieser komplexen Verben vor, wobei das Nomen aus der Objektposition durch 'Move alpha' ins Verb versetzt wird. Dies wurde jedoch kritisiert, unter anderem von Di
Nomeninkorporation

Sciullo \& Williams (1987), die für eine eigenständige lexikalische Formierung eintreten. Auf diese Frage möchte ich jedoch hier nicht näher eingehen.

\section{Nomeninkorporation im Fering}

Für eine allgemeine Charakterisierung des Friesischen wäre es natürlich interessant; nachzuforschen, ob auch in anderen friesischen Dialekten Nomeninkorporation vorkommt, und im Rahmen des Föhrer Symposiums liegt es auf der Hand, das Fering diesbezüglich zu untersuchen. Dies ist für einen Nicht-Feringer selbstverständlich keine leichte Aufgabe, und darüber hinaus hat eine Untersuchung der Nomeninkorporation generell ihre eigenen Schwierigkeiten. In den föhrerfriesischen Grammatiken wird sie nicht erwähnt, und in gedruckten mundartlichen Texten begegnet man ihr auch relativ selten.

Die einzige, die sich meines Wissens explizit zur Nomeninkorporation im Fering geäußert hat, ist Ebert (1989); sie schreibt denn auch konsequent die Kombination von Objekt und Verb als ein Wort. Leider gibt sie keine formalen Argumente, und möglicherweise ist bei einigen ihrer Beispiele auch gar keine Inkorporation im Spiel. Inkorporation ist nämlich nicht immer leicht zu erkennen. Verantwortlich dafür ist eine konkurrierende (Dyk 1992) syntaktische Konstruktion mit einem selbständigen kahlen Objekt, das sich nur in finiten Hauptsätzen leicht unterscheiden läßt:

(10) a. Hy drinkt wetter ('Er trinkt Wasser')

b. Hy wetterdrinkt

In diesen Beispielen aus dem Frysk stellt (10a) die syntaktische Konstruktion dar. Leider kommt die Verwendung in finiten 
Kontexten nicht häufig vor. In allen anderen syntaktischen Kontexten, die nicht als finite Hauptsätze gekennzeichnet sind, steht das Objekt unmittelbar vor dem Verb, und zwar in beiden Konstruktionen. Hier das Beispiel eines finiten untergeordneten Satzes:

(11) a. ... dat er wetter drinkt

b. ... dat er wetterdrinkt

Ich habe die beiden Konstruktionen ganz bewußt orthographisch voneinander unterschieden, doch aus Gründen, die nichts mit dieser Konstruktion zu tun haben, ist es vorstellbar, daß jemand, der mit dieser Erscheinung weniger vertraut ist, auch weniger * konsequent sein könnte, vor allem wenn er in einer schriftlichen Tradition steht, die keine Nomeninkorporation kennt. Ansonsten gibt es keine formalen Unterschiede, nur phonetisch läßt sich eine Verschiedenheit feststellen, die allerdings äußerst subtil ist. In beiden Varianten wird das Objekt stärker betont als das Verb, einen noch stärkeren Akzent erhält jedoch das Objekt beim inkorporierenden Verb. Dies wurde mir in Bezug auf das Fering so bestätigt.

Bei Stoffnamen oder im allgemeinen bei nicht-zählbaren Objekten wie wetter ('Wasser') ist es jedoch sehr schwer, auf syntaktische Selbständigkeit zu schließen, d.h. zu bestimmen, ob oder ob nicht inkorporiert wird. Dies gilt gleichermaßen für das Frysk und das Fering. Im Frysk ist die Lage bei zählbaren Objekten jedoch einfacher. Die syntaktische Konstruktion mit einem selbständigen Objekt zeigt sich hier meistens in der Form eines kahlen Plurals (12), weil wir bei der Inkorporation entweder ein Schwa als Zwischenlaut oder gar keine Endung vorfinden (13):

(12) a. ... dat er biten dolt

('daß er Rüben ausgräbt') b. ... dat er jerappels dolt ('daß er Kartoffeln ausgräbt')

(13) a. ... dat er bitedolt ... dat er bytdolt

b. ... dat er jerappeldolt

Im Fering scheint die Lage bei zählbaren Nomina weniger durchsichtig zu sein. Ebert teilt mit, daß Inkorporation bei der uunt-Konstruktion obligatorisch ist. Jedoch erscheinen hier, anders als im Frysk, auch Pluralendungen, zum Beispiel bei Ebert (1989:295):

(14) Hat as uunt höözenstoopin

eerdaaplerskelin

anhenrupin

kuukenbaagen

brekenklütjin

('Sie ist am Strümpfestopfen')

Kartoffelnschälen')

Entenrupfen')

Kuchenbacken')

Hosenflicken')

Laut Ebert ist der Numerus hier semantisch leer, was tatsächlich auf Inkorporation hinweist. Dennoch führt das Wurdenbuk (Wilts 1986) sub voce skele Objekt und Verb getrennt auf:

(15) Det foomen as uun't eerdaapler skelin

('Das Mädchen ist am Kartoffeln schälen')

Noch komplizierter ist die Lage bei den $t u$-Infinitiven. In den meisten Fällen, die ich angetroffen habe, findet man eine Pluralendung und überwiegend Getrenntschreibung. Nur Ebert 


\section{Siebren Dyk}

schreibt Objekt und Verb auch hier zusammen, obwohl sie (S. 301) mitteilt, daß Inkorporation bei den $t u$-Infinitiven fakultativ ist. Übrigens besteht hier ein Unterschied zum Frysk: Dort wird in dem vergleichbaren Kontext sogar obligatorisch inkorporiert. Eine kleine Auswahl dessen, was ich bei $t u$-Infinitiven angetroffen habe, folgt hier:

(16) tu Robben kloppin (Arfsten 1896:14)

('zu Robben schlagen')

tu Trossen splassin (Arfsten 1896:37)

('zu Trossen spleißen')

tu boten pregin (Wilts 1986 s.v. bot)

('zu Butte stechen')

tu beien plookin (Wilts 1986 s.v. bei)

('zu Beeren pflücken')

tu höözenstoopin (Ebert 1989:300)

('zu Strümpfestopfen')

tu hanenfulrin (Ebert 1989:309)

('zu Hühnerfüttern')

Alles in allem scheint es der Deutlichkeit halber am zweckmäßigsten zu sein, wenn wir uns auf diejenigen Fälle beschränken, bei denen das Objekt nicht in einer gleichen Form syntaktisch selbständig vorkommen kann. Diese Fälle finden wir nämlich auch im Fering. Hier folgt eine Auswahl:

(17) tu taag ufbinjen (Wilts 1986 s.v. ufbinj)

('zu Reetdach abdecken' (Reet bündeln))

tu busem besen (Wilts 1986 s.v. besä)

('zu Stall betrachten')

uunt welklütjin (Ebert 1989:295)

('am Fahrradflicken')

uunt jakpreglin (Ebert 1989:320)

('am Jackestricken')

\section{Nomeninkorporation}

... kön jam wirem greew (Bahns u.a. 1978:47)

('... könnt ihr Wurm graben')

mä tanebuum uftaaklin (Wilts 1986 s.v. uftaakle)

('mit Tannenbaum plündern')

sin Briafskriiwen (Arfsten 1958:47)

('sein Briefschreiben')

piip greew (Wilts 1986)

('Kleigraben graben' (in den Marschweiden))

gredpluuge (Wilts 1986)

('Graslandpflügen')

greefmaage (Wilts 1986)

('Grabausheben')

Von diesen Beispielen ausgehend, muß man zu dem Schluß kommen, daß das Objekt hier Bestandteil eines Wortes ist. Jedenfalls tritt ein singuläres Objekt ohne Determinator in den mir bekannten germanischen Sprachen nicht auf, so daß ich annehme, daß dies auch für das Fering gilt:

(18) ${ }^{*}$ Hat pregelt jak

Eine solche Form ist sozusagen nur zu 'retten', wenn man sie als Teil eines Kompositums betrachtet. Beispiele wie (17) sind besonders geeignet, den Wortcharakter der Kombination zu beweisen. In Dyk (1992) habe ich in Bezug auf das Frysk eine Reihe von Tests vorgestellt, die im allgemeinen zeigen, daß aus einem Wort kein Element extrahiert werden kann. So kann (18) als Beispiel gelten, wenn man von einer dem Fering zugrundeliegenden SOV-Folge ausgeht:

(19) a. Hat [pregelt $]_{i}$ at jak $t_{i}$

b. *Hat [pregelt] ${ }_{\mathrm{i}}$ jak $\mathrm{t}_{\mathrm{i}}$ 
Zwei andere Tests beziehen sich auf die Versetzung des Objekts ('Scrambling') und das Weglassen des Verbs. Solche Prozesse sind nur möglich, wenn das Objekt eine syntaktisch selbständige Phrase bildet:

(20) a. Ik skal daaling [at jak $]_{\mathrm{i}}$ ei ti pregle

('Ich werde heute die Jacke nicht stricken')

b. *Ik skal daaling [jak $]_{\mathrm{i}}$ ei ti pregle

(21) a. Ik skal daaling at brek pregle an Mam at jak pregle

('Ich werde heute die Hose stricken und Mutter die Jacke')

b. *Ik skal daaling brekenpregle an Mam jak pregle

Ich möchte also auf Grund der Mitteilungen Eberts und auch der obengenannten mehr oder weniger deutlichen Fakten die Schlußfolgerung ziehen, daß es im Fering tatsächlich Nomeninkorporation gibt. Dabei ist es meines Erachtens nur natürlich anzunehmen, daß auch die nicht-zählbaren Nomina und die Nomina mit einer Pluralmarkierung mitinkorporieren können.

Eine andere Frage ist, ob die inkorporierten Formen im ganzen verbalen Paradigma auftreten können. Besonders die finiten Formen sind hier ein Problem. Im Frysk sind solche Formen nicht sehr häufig, jedoch auch nicht ausgeschlossen, insbesondere wenn sie mit durativen Bestimmungen 'beschwert' werden. In meiner Datensammlung gibt es mehr als 60 Beispiele finiter Nomeninkorporationsformen aus frysken Texten. Möglicherweise besteht hier ein Unterschied zum Fering. Mein Informant, für den die form piipgreew, 'Abzugsgräben auf einer Fenne ausheben' durchaus akzeptabel ist, weigert sich jedoch, eine finite Form dieses Verbs zu akzeptieren, auch wenn diese 'beschwert' ist: 1

(22) *Wi piipgreew al a hiale dai
Trotzdem frage ich mich, ob diese Erschwernis die Möglichkeit zur Nomeninkorporation einschränkt. Laut Ebert (1989:308) kann 'die einfache Form der meisten Verben, die Tätigkeiten benennen, (...) nicht das aktuelle Präsens ausdrücken'. Sie nennt unter anderem die Beispiele:

\section{(23) *wi slaachti ('wir schlachten') *ik moolki ('ich melke')}

Es würde den Rahmen sprengen, darauf weiter einzugehen, aber meiner Überzeugung nach besteht in. diesem Bezug kein wesentlicher Unterschied zwischen den intransitiven Verben mit und ohne solchen Inkorporation. Wenn ${ }^{*} i k$ moolki unakzeptabel ist, läßt sich daraus folgern, daß zum Beispiel auch *ik kümoolki ('ich Kuhmelke') nicht akzeptabel ist. Meiner Meinung nach sind es eigensprachliche Bedingungen, die im Fering Nomeninkorporation in finiten Kontexten ausschließen; mit der Möglichkeit oder Unmöglichkeit zur Nomeninkorporation selber hat dies nichts zu tun.

\section{Die historische Ursache}

Unsere Annahme, daß es im westerlauwersschen und Föhrer Friesischen so etwas wie eine Nomeninkorporation gibt, hat sich m.E. weitgehend bestätigt. Auch wenn dies allein schon als linguistische Erkenntnis oder als Beitrag zur Bestimmung der Eigenart des Friesischen möglicherweise nicht unwichtig ist, so scheint mir dennoch die Frage noch interessanter, warum es das Phänomen der Nomeninkorporation überhaupt gibt. Warum verfügt das Friesische - auf jeden Fall das Frysk und das Fering - im Gegensatz zu den anderen germanischen Sprachen über die Möglichkeit der Nomeninkorporation? Eine strukturelle Erklärung 
scheint sich hier zunächst anzubieten. Dies bedeutet, daß wir nach einer strukturellen Eigenschaft suchen müssen, die es im Friesischen, jedoch nicht in den anderen germanischen Sprachen gibt. Solch eine Eigenschaft ist das Vorkommen zweier Infinitivformen im Friesischen, deren Verwendung ich zunächst darlegen möchte.

Schon in der ersten amringer Grammatik (Johansen 1862) werden zwei Infinitivformen unterschieden, allerdings ist bei Johansen die Reihenfolge gerade umgekehrt als in späteren Werken, in denen man von einem Infinitiv I, mit einem Suffix $-i$ oder einem $\emptyset$-Suffix, und einem Infinitiv II mit einem Suffix -en oder - $n$ spricht. Wir bekommen also (Århammar 1969:119):

$\begin{array}{lll}\text { (24) Infinitiv I } & \text { Infinitiv II } & \\ \text { swaari } & \text { swaarin } & \text { 'antworten' } \\ \text { feel } & \text { feelen } & \text { 'fühlen' } \\ \text { fu } & \text { fun } & \text { 'bekommen' }\end{array}$

Am ausführlichsten behandeln Schmidt-Petersen \& Craigie (1928:32) die syntaktischen Bedingungen für das Vorkommen des Infinitivs II. Sie nennen insgesamt fünf Konstruktionen (die Glossen sind von den Autoren übernommen worden):

(25) (i) As a Substantive after the Article, e.g. det plugin.

(ii) Absolutely in Questions and Answers, e.g. wat hält beder, seien of plokin?

(iii) After certain definite verbs, particularly those of motion and perception. satten bliw bellen hiar 'to remain sitting' gungen se 'to hear bark' 'to go to see'

(iv) After nant uss - nothing but, only, merely, e.g. hi dä nant üss spellin 'he does nothing but play'
(v) After the preposition $t u$ 'when it equals Latin 'ut'. e.g. $\begin{array}{ll}\text { tu plugin } & \text { 'in order to plough' } \\ \text { tu rawen } & \text { 'in order to rest' }\end{array}$

Mein an Hand der feringer Texte gewonnener Eindruck ist der, daß diese Beschreibung nicht ausreicht, um alle Vorkommen des Infinitivs II im Fering zu erfassen. Doch ist dies nicht der Ort, um hierauf weiter einzugehen. Wichtiger ist, daß man aus diesen fünf Fällen ableiten kann, daß der Infinitiv II vor allem in nominalen Umgebungen vorkommt. Das ist auch die Folgerung Willem Vissers, der das Vorkommen des Infinitivsuffixes -en im Frysk untersucht hat (Visser 1989). Er kommt zu dem Schluß, daß das -en-Suffix, jedenfalls was den durch ihn untersuchten nominalen Infinitiv anbelangt, im Lexikon angehängt wird; es sollte, mit anderen Worten, nicht zur Flexion, sondern zur Wortbildung gehören.

Welche mögliche Verbindung besteht nun zwischen dem nominalen Infinitivsuffix -en und dem Phänomen Nomeninkorporation, also den komplexen Verben mit einer [NV]-Struktur? Einen ersten Erklärungsansatz bietet das Wortbildungsprinzip der synthetischen Komposition, also die 'Zusammenbildung'. In diesem Zusammenhang ist eines der bekanntesten Affixe das Suffix -er. Es bezeichnet den Träger des Geschehens (Nomina agentis) und kommt in vielen germanischen Sprachen vor:

(26) Kaffeetrinker (Deutsch)

coffee drinker (Englisch)

koffiedrinker (Holländisch)

kofjedrinker (Frysk)

kofedranker (Fering)

Dieses Wort besteht aus drei Elementen: Kaffee, trink und -er. Für die Struktur dieses Worts gibt es logischerweise drei Möglichkeiten: 
(27) a

\begin{tabular}{|c|c|c|}
\hline \multicolumn{3}{|c|}{$\mathrm{N}$} \\
\hline & ] & $\backslash$ \\
\hline 1 & 1 & 1 \\
\hline 1 & 1 & 1 \\
\hline & $\mathrm{V}$ & $N$ \\
\hline 1 & 1 & 1 \\
\hline
\end{tabular}

b.

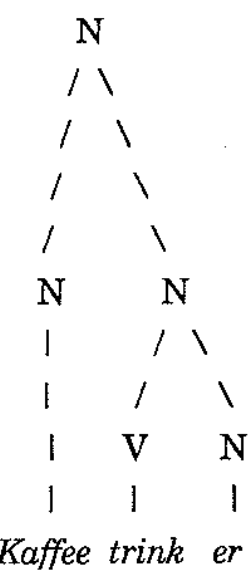

c.

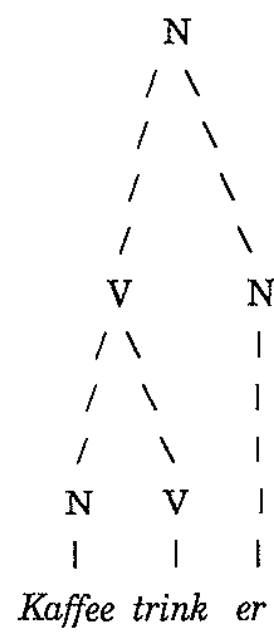

Möglichkeit (27a) wird wohl nicht viel Anhänger haben, und zwar weil die Struktur nicht binär ist (die 'Binary Branching Hypothesis', siehe z.B. Scalise (1984:146)). Anders ist es mit der Struktur (27b), die im Anfang der achtziger Jahre vor allem von amerikanischen Linguisten propagiert worden ist (Allen 1978, Selkirk 1982, Lieber 1983). Diese Auffassung ist jedoch von Rudolph P. Botha (1981; 1984) kritisiert worden, meines Erachtens aus. guten Gründen. Das Wichtigste dabei ist die semantische Interpretation, die, anders als bei primären NN-Komposita, eindeutig festliegt und die sich in der Struktur widerspiegeln sollte, vgl. Botha (1984:110);

\section{(28) Compositionality Condition}

The morphological representation assigned to a complex word must provide the labelled bracketing necessary for the specification of its semantic interpretation.

Ein Kaffeetrinker ist also 'jemand, der Kaffee trinkt', und nicht primär 'ein Trinker, der auf die eine oder andere Weise etwas mit Kaffee zu tun hat', wie Struktur (27b) darstellt. ${ }^{2}$

Auf die eigentliche Ableitung von Strukturen wie (27c) will ich hier nicht eingehen, am wichtigsten erscheint mir, daß solche Wortstrukturen in der Sprache vorkommen. Interessant in der Struktur $(27 \mathrm{c})$ ist der linke untere Teil, also:

$$
\begin{array}{ll}
\mathrm{V} \\
/ \mathrm{N} \\
\mathrm{N}
\end{array}
$$

Wir sehen, daß dies auch genau die Struktur ist, die denjenigen Verben zugewiesen werden muß, die durch Nomeninkorporation entstanden sind. Meine These ist nun, daß das Suffix en des Infinitivs II in vergleichbarer Weise wie das obengenannte -er 


\section{Siebren Dyk}

gewirkt hat und Strukturen wie (27c) gebildet hat. Ersetzen wir dort $e r$ durch en und die hochdeutschen Wörter durch friesische, so erhalten wir das richtige Resultat. In abstracto also:

$$
\begin{array}{ccc}
\mathrm{N} & \\
/ & 1 \\
/ & 1 \\
\mathrm{~V} & 1 \\
/ & 1 & 1 \\
\mathrm{~N} & \mathrm{~V} & e n
\end{array}
$$

Wahrscheinlich ist es dabei nicht ohne Bedeutung, daß man, wie wir gesehen haben, dem Suffix -en nominale Eigenschaften zuweisen kann. Meines Wissens sind es immer Wortbildungsaffixe, díe derartige Strukturen wie (27c) generieren, zum Beispiel im Frysk die nominalisierenden Suffixe -er und -erij und das adjektivische -erich. Man erhält also kofjedrinker, kofjedrinkerij und kofjedrinkerich. Solche Operationen sind mir nicht bekannt von Flexionsaffixen; die die Kategorie des Wortes, an das sie angehängt werden, nicht ändern können. Man kann also nicht von dem Infinitiv I, der meistens mit dem Suffix $-e$ gebildet wird, erwarten, daß dieser in der Lage ist, Strukturen wie $(27 \mathrm{c}) \mathrm{zu}$ bilden, weil mit Visser (1989) anzunehmen ist, daß das Infinitivsuffix $-e$ zur Flexion gehört.

Eine entsprechende Begründung gilt für das Niederländische, das nur eine Infinitivform kennt, von der Hoekstra (in diesem Band) angenommen hat, daß diese nicht für eine Kategorie spezifiziert ist. Im Niederländischen ist das Infinitivsuffix also nicht 'stark' genug, um Strukturen wie (30) zu bilden. Im Niederländischen findet man denn auch keine Nomeninkorporation. Mutatis mutandis gilt dasselbe für das Deutsche und die skandinavischen Sprachen, die auch nur über ein einziges Infinitivsuffix verfügen. Auch da gibt es keine Nomeninkorporation. Demgegenüber ist die Sachlage im Englischen

\section{Nomeninkorporation}

etwas komplizierter. Darauf komme ich noch später zu sprechen.

Zum besseren Verständnis und um nicht vorauszueilen, möchte ich noch einmal festhalten, daß das Suffix -en des friesischen Infinitivs II in Zusammenbildungsstrukturen wie (27c) eine Rolle spielt. Wir konnten aber auch zeigen, daß Verben mit Nomeninkorporation im Friesischen sich nicht nur auf Formen mit diesem Suffix beschränken, sondern daß sie im Prinzip innerhalb des ganzen verbalen Paradigmas vorkommen. Dabei muß allerdings eine Struktur wie (29) vorliegen. Nun stellt sich allerdings das Problem, wie wir von der in (30) dargestellten Struktur zu der Struktur von (29) gelangen können.

Zunächst müssen wir hierfür das Suffix wieder 'loswerden'. Theoretisch gesehen ist das möglich durch einen Prozeß, der im Englischen als 'backformation' bzw. im Deutschen als 'Rückbildung' oder auch 'retrograde Ableitung' bezeichnet wird. Adams beschreibt dies mit den Worten: 'the more complex word comes first and then some element is subtracted from it, resulting in a pair of words which conforms with a base $\rightarrow$ derived pattern already existing' (Adams 1973:105). Das bereits existierende Wortbildungsmuster ist hier selbstverständlich die normale Ableitung des Infinitivs II mit einem verbalen Stamm als Grundlage und einem angehängten Suffix -en. Man kann sich also die Rückbildung so vorstellen, daß der Sprachbenutzer aus dieser regelmäßigen Korrelation des verbalen Grundwortes und des Infinitivs II im allgemeinen auf die Existenz einer Grundform ohne Suffix schließt, also:

(31) $\left[[N V]_{V}\right.$ en $] \rightarrow[N V]_{V}$

Natürlich taucht nun die Frage auf, warum ein Suffix wie das Nomina agentis bildende -er nicht an der Basis einer Rückbildung stehen kann. ${ }^{3}$ Hierbei ist zu beachten, daß es einen wichtigen Unterschied zwischen -en und -er gibt. Bildungen mit 
-er sind nämlich immer auf typisch nominalen Positionen in der Satzstruktur zu finden. Semantisch haben sie immer eine Person und daher ein Argument als Referenten. Demgegenüber ist -en mit einem Januskopf zu vergleichen. So kann es nämlich sein, daß -en auch eine nominale Umgebung bevorzugt, das sind dann aber höchstens Nominalisierungen einer Handlung. Die -enAbleitungen referieren daher nicht nach Argumenten, sondern nach Prädikaten. Sie können zum Beispiel nicht pluralisiert werden, auch scheint es mir nicht unerheblich zu sein, daß sie nach traditionellem Verständnis als Infinitive gekennzeichnet werden.. Man könnte also behaupten, daß sie zum verbalen Paradigma gehören. So ist zu erklären, da $\beta$ sich in dieser Situation eine verbale Grundform leicht auf die ursprüngliche Konstruktion zurückführen läßt.

Ich nehme an, daß diese Rückbildung ein diachronischer Prozeß gewesen ist. Ein Argument dafür bildet das Gronings, ein niedersächsischer Dialekt, der in der niederländischen Provinz Groningen, östlich von westerlauwerssch Friesland, gesprochen wird. Auch im Gronings kommt Nomeninkorporation vor (Schuurmans \& Wierenga 1986). Das Gronings verfügt heutzutage nur über ein Infinitivsuffix, und zwar -en (Ter Laan 1953:68). Das Vorkommen der Groninger Nomeninkorporation kann meines Erachtens nur dadurch erklärt werden, daß diese Gegend im Mittelalter noch zum friesischen Sprachraum gehört hat und daß der neue Dialekt diese Wortbildungsmöglichkeit vom Substrat übernommen hat. Wäre die Rückbildung nur synchronisch bedingt, ließe sich die Existenz der Nomeninkorporation im Gronings nicht erklären.

Ein zweites Argument für die Behauptung, die Rückbildung sei diachronisch bedingt, ist rein theoretisch. Synchronisch gesehen glaubt man, daß diese Art der Wortbildung sehr markiert und außerdem ein Wort-zu Wort-Prozeß sei, der naturgemäß viele Ausnahmen kennt. Im Frysk jedenfalls hat sich gezeigt, daß die
Inkorporation sehr produktiv ist und daß mühelos neue komplexe Verben gebildet werden können. Dazu kommt, daß Rückbildungsprodukte oft Abweichungen in ihrem Paradigma haben, zum Beispiel bei den starken Verben. So hat das niederländische zuigen als Präteritum die Form zoog, aber das Rückbildungsverb stofzuigen ist im Präteritum stofzuigde, also ganz regelmäßig konjugiert. Im Frysk dagegen sind solche Paradigmaabweichungen nicht zu finden: die Konjugation des komplexen Verbs ist dieselbe wie die Konjugation des einfachen Verbs. So hat zum Beispiel das Verb lêze 'lesen' als Perfektpartizip das starke lezzen. Dieser Form begegnen wir auch im komplexen boekjelêzen und nicht dem schwachen *boekjelêsd.

Man muß also wohl konkludieren, daß die Rückbildung synchron nicht mehr aktiv ist und daß die Bildung dieser komplexen Wörter von einem normalen produktiven Worbildungsmechanismus übernommen worden ist. Angesichts des Resultats kann man sich überhaupt fragen, ob es hier jemals eine konventionelle Wort-zu-Wort-Rückbildung gegeben hat oder ob die Rückbildung nicht eher auf einer strukturellen Ebene anzusiedeln wäre, wie wir sie in (31) schon vorgestellt haben. In diesem Fall müßten wir die Ursache einer derartigen strukturellen Rückbildung von neuem suchen in den speziellen 'doppelköpfigen' Eigenschaften des Suffixes -en, das viel näher beim verbalen Paradigma steht als zum Beispiel das 'einköpfige' nominale -er.

Zum Schluß möchte ich noch eine zusätzliche Begründung der hier vorgestellten These anführen, daß im Friesischen das Infinitiv II - Suffix letzten Endes verantwortlich ist für die Fähigkeit zur Nomeninkorporation. Dazu müssen wir unseren Blick über die Nordsee nach England richten. Im Englischen existiert nämlich neben dem ausgangslosen normalen Infinitiv eine weitere Form mit dem Suffix -ing, die meistens 'gerund' genannt wird. Es läge nahe, anhand dieses Terminus und unterstützt durch die Aussagen Århammers (1964:119) zum Amringer und Sytstra \& Hofs (1925:134) zum westerlauwersschen 


\section{Siebren Dyk}

Friesisch - daß nämlich das -en-Suffix aus einem altfriesischen Gerundiumsuffix entstanden sei - den Schluß zu ziehen, daß wir es hier mit demselben Phänomen zu tun haben. Daß eine derartige Schlußfolgerung zu einfach wäre, verdeutlicht der Übersichtsartikel von Jack (1988) über die Entstehung des Gerunds. Dennoch kann man sagen, daß -en und -ing vergleichbare Eigenschaften haben: Beide stehen im verbalen Paradigma neben einem 'primären' Infinitiv, und beide Formen werden auch selektiert in nominalen Umgebungen (siehe u.a. Pullum 1991). Aber -ing hat noch eine weitere Eigenschaft. Im Englischen ist gerade dieses ing, neben -er und -ed, eines der drei Suffixe, die sehr produktiv Zusammenbildungen bilden können. Beispiele sind mountainclimbing, cakebaking usw. Es ist also nicht unwahrscheinlich, - daß das vergleichbare friesische Suffix gleiche Möglichkeiten gehabt hat, um Zusammenbildungen zu produzieren.

Leider stellt sich damit ein neues Problem, nämlich warum man im Englischen keine Nomeninkorporation findet, obwohl die Bedingungen denen im Friesischen anscheinend entsprechen. Ist dies nur zufällig? Eine naheliegende Erklärung für das Fehlen der Rückbildungen im Englischen könnte sein, daß -ing weiter vom übrigen verbalen System entfernt ist als das friesische en-Suffix. Aber dies scheint, soweit ich das beurteilen kann, nicht der Fall zu sein. Was die Plazierungsmöglichkeiten betrifft, könnte man sogar eher vom Gegenteil sprechen. Entsprechend meinen Ausführungen zu den -er-Derivaten, sehe ich dann auch keinen Grund, eine Rückbildung bei -ing-Ableitungen auszuschließen.

Um einer Lösung des Problems näherzukommen, warum das Friesische inkorporiert und das Englische nicht, sollten wir die englischen Fakten eingehender betrachten. Denn die Aussage, das Englische habe keine Nomeninkorporation, ist nicht völlig zutreffend. Es gibt nämlich komplexe Verben mit NV-Zusammensetzung, wie to aircondition oder to babysit usw. Dabei muß aber gesagt werden, daß diese Bildung nicht produktiv ist, ganz im
Gegensatz zum Friesischen. Die Situation scheint so zu sein, daß, während die NV-Zusammenbildung mit -ing ganz normal ist, die Rückbildung zum komplexen Verb bei sehr vielen dieser Formationen blockiert ist. Man hat also meat-eating und taxpaying, aber kein *to meat-eat oder *to tax-pay. Shimamura (1983) hat in diesem Zusammenhang zwei sehr interessante Beobachtungen gemacht, für die er leider keine Erklärung gibt. Die erste, in Anschluß an Allen (1978), ist die, daß im allgemeinen nur diejenigen -ing-Formationen zu einem komplexen Verb zurückgebildet werden können, die keine kompositionelle semantische Beziehung zwischen dem Nomen und dem Verb zeigen; die Bedeutung ist hier also in einem mehr oder weniger starken Maße spezialisiert oder idiomatisiert, und man kann annehmen, daß derartige Verbindungen zwischen Verb und Nomen lexikalisiert sind.

Die zweite Beobachtung ist, daß bei den Beispielen, bei denen man dennoch von einer kompositionellen Beziehung reden kann, das Nomen nicht in einer 'First Sister'-verbindung mit dem Verb steht. Das 'First Sister Principle' stammt aus einem Artikel von Roeper \& Siegels (1978:208) über englische Zusammenbildungen und lautet:

(32) 'All verbal compounds are formed by incorporation of a word in first sister position of the verb'.

Shimamura (1983:274) nennt die Beispiele

(33) spoon-feeding (feeding someone with a spoon) cf. spoon-feed speed-reading (reading something with speed) cf. speed-read test-flying (flying something as a test) cf. test-fly

Die 'inkorporierten' Nomina besetzen hier in der Tat nicht die 'erste Schwester' Position; diese wird hier eingenommen durch 


\section{Siebren Dyk}

eine NP, die in (33) mit someone oder something angedeutet ist. Aber was hier weiterhin ins Auge fällt, ist, daß die Nomina in diesen Fällen als ein Adjunkt beim Verb fungieren und nicht als ein Argument (siehe die Hinweise, daß Instrumente wie obengenanntes spoon auch als Adjunkte fungieren, bei Jackendoff 1990:281). Auch Allen (1978) hat schon darauf hingewiesen, daß die Zusammenbildung hier Lücken aufweist; zum Beispiel hat man spoonfed, aber nicht *knifefed und daher auch kein *to knifeféed. Auch hier könnte man also von einer Art Lexikalisierung sprechen. Außerdem muß man konkludieren, daß die Nomina, wenn sie in einer freien syntaktischen Verbindung auftreten, als Adjunkte nicht durch das Verb regiert werden.

Die freie syntaktische Verbindung scheint mir in diesem Zusammenhang relevant zu sein. Wie bekannt, ist das Englische eine SVO-Sprache, und daher regiert das Verb im Englischen nach rechts. So steht zum Beispiel das Objekt auch rechts vom englischen Verb. Wir haben also:

\section{(34) V NP}

Aber was geschieht im korrespondierenden Fall, in dem das komplexe Verb aus einer -ing-Zusammenbildung rückgebildet wird? Das Nomen steht dann noch immer links vom Verb, was auch nicht anders möglich ist, weil in der Morphologie das Haupt immer rechts zu finden ist (Williams' 1981 Right Hand Head Rule):

(35) $\mathrm{NV}$

Meines Erachtens liegt hier die Erklärung für die entscheidenden Beschränkungen der Rückbildung im Englischen. Bei den transparenten Verbindungen eines Nomens und eines Verbs wird die Folge Nomen - Verb (35) ausgelöscht durch die viel

\section{Nomeninkorporation}

grundlegendere Folge Verb - Nominalphrase (34) in der Syntax. Nur in zwei Fällen ist dieser syntaktische Zwang nicht möglich. Zum einen, wenn die Kombination eines Nomens und eines Verbs lexikalisiert ist, also in den Fällen ohne kompositionelle Bedeutung. Diese bilden sozusagen eine lexikalische Einheit, die die Syntax, die ihrem Wesen nach mehr als die Morphologie an transparenten Beziehungen orientiert ist, nicht mehr zu beeinflussen vermag. Zum anderen, wenn das Nomen syntaktisch nicht vom Verb regiert ist, also bei Adjunktverhältnissen (und wie wir gesehen haben, könnte auch hier eine Art Lexikalisierung im Spiel sein).

$\mathrm{Daß}$ die englische syntaktische SVO-Folge tatsächlich im Stande ist, eine morphologische OV-Folge zu verdrängen, kommt in einer Untersuchung der englischen Kindersprache (Clark, Hecht \& Mulford 1986) besonders gut zum Ausdruck. Befragt nach Agens- und Instrumentnamen, zeigte es sich, daß Kinder, auch wenn sie das Suffix schon angelernt hatten, noch lange eine Verb Objekt -Folge verwendeten. Man begegnet so Formen wie

\section{(36) *dryer-hair}

Erst im Alter von fünf, sechs Jahren verändert sich diese Form in die bei Erwachsenen gebräuchliche Form hairdryer. Die Untersucher schreiben die Abweichung einer Generalisierung zu, die die Kinder machen:

(37) 'All Verb + Obj combinations have canonical predicate order'.

Es ist anzunehmen, daß diese Generalisierung noch stärker wirkt, wenn die morphologische Bildung von Verb und Objekt ein wirkliches Prädikat designiert, wie es bei den komplexen Verben der Fall ist. Demgegenüber unterscheidet sich das Friesische grundlegend. Wie in den übrigen westgermanischen Sprachen 
liegt im Friesischen eine syntaktische SOV-Folge zugrunde nach dem Muster (38)

\section{(38) NP V}

Weil so die syntaktische Gegenkraft fehlt, ist im Friesischen die Rückbildung nicht blockiert worden. Aus diesem Grunde ist die Bildung der komplexen NV-Verben frei ${ }^{4}$ möglich geworden, so daß man behaupten kann, daß das Friesische eine nomeninkorporierende Sprache ist. Fazit ist, daß es diese Eigenschaft meines Erachtens nur der Tatsache zu verdanken hat, daß die Sprache über ein spezielles Infinitivsuffix verfügt, das die spezifischen Grundbedingungen zur Bildung der hier behandelten komplexen Verben möglich gemacht hat.

\section{Fryske Akademy \\ Doelestrjitte 8 \\ NL-8911 DX Ljouwert}

\section{Anmerkungen}

* Ich danke Volkert F. Faltings für seine Hilfe beim Fering und Jarich Hoekstra und Willem Visser für ihre kritische Lesung einer früheren Version. Selbstverständlich ist nur der Autor verantwortlich für die Fehler.

1. In der Diskussion im Anschluß an den Symposiumsvortrag wurden die Schwierigkeiten bei der Inkorporation bei finiten Verben von anderen Sprechern bestätigt.

2. Struktur (27c) entspricht nicht exakt der Bothas. Seine Theorie leitet die Zusammenbildung von syntaktischen
Tiefenstrukturen ab, daher weichen die Kategoriebezeichnungen ab. Bei ihm sollte (27c) so etwas sein wie $\left[\left[[\text { Kaffee }]_{N P}[\text { trink }]_{\mathrm{V}}\right]_{\mathrm{VP}} \text { er }\right]_{\mathrm{N}}$ (Botha 1981:53). Für meine Beweisführung macht das jedoch nichts aus.

3. Ganz und gar ausgeschlossen ist die Rückbildung hier nicht. Zum Beispiel wird für das Niederländische angenommen, daß stofzuigen 'staubsaugen' entstanden ist aus stofzuiger (Booij 1990). Aber davon gibt es nur ein paar isolierte Fälle.

4. Auch diese Freiheit ist eingeschränkt, aber dennoch kann man sagen, daß die Bildung der komplexen Verben sehr produktiv ist, vergleichbar mit der Produktivität der Zusammenbildungen der Nomina agentis mit dem Suffix -er. Die Einschränkungen bedürfen einer separaten Behandlung, die hier nicht geleistet werden kann.

\section{Bibliographie}

Adams, V. 1973. An introduction to modern English wordformation. London: Longman.

Allen, M. 1978. Morphological Investigations. Ph.D. dissertation, University of Connecticut.

Arfsten, A.J. 1896. [A.J. Arfsten sin Düntjis, ütjdenn fan Dr. Otto Bremer]. Halle: Max Niemeyer.

Arfsten, R. 1958. Mamenspriik. Leesebuk för Fehr an Oomram. Wyk auf Föhr: Nordfriesischer Verein für Heimatkunde und Heimatliebe.

Århammar, N. 1969. 'Die Amringer Sprache'. In: M. \& N. Hansen (eds.) Amrum - Geschichte und Gestalt einer Insel (2. Aufl.). Itzehoe-Münsterdorf, Hansen \& Hansen.

Bahns, P. u.a. 1978. Fering.Öömrang Skuulbuk. Bräist/Bredstedt: Nordfriisk Instituut. 
Baker, M. 1988. Incorporation. A theory of grammatical function changing. Chicago \& London: The University of Chicago Press.

Booij, G. 1990. 'Complexe werkwoorden en de niveauordeningshypothese'. Spektator 19:234-244.

Botha, R.P. 1981. 'A base rule theory of Afrikaans compounding'. In M. Moortgat, H. van der Hulst \& T. Hoekstra (eds.). The scope of lexical rules. Dordrecht: Foris, 1-77.

Botha, R.P. 1984. Morphological Mechanisms. Lexicalist analysis of synthetic compounding. Oxford etc.: Pergamon Press.

Clark, E.V., B.F. Hecht \& R.C. Mulford. 1986. 'Coining complex compounds in English: affixes and word order in acquisition'. Linguistics 24:7-29.

DiSciullo, A.M. \& E. Williams. 1987. On the definition of the word. Cambridge (Mass.): The MIT-Press.

Dyk, S. 1992. 'Aspekt en nomenynkorporaasje'. Philologia Frisica anno 1990. Ljouwert:Fryske Akademy.

Ebert, K. 1989. 'Aspektmarkierung im Fering (Nordfriesisch) und verwandten Sprachen'. In W. Abraham \& Th. Jansen (eds.). Tempus. Aspekt - Modus. Die lexikalischen und grammatischen Formen in den germanischen Sprachen. Tübingen: Max Niemeyer, 293-322.

Hoekstra, J. (in diesem Band). 'Fering $t u$-infinitives, North Sea Germanic syntax and Universal Grammar'.

Jack, G. 1988. "The origins of the English gerund'. NOWELE 12:15-75,

Jackendoff, R. 1990. Semantic structures. Cambridge (Mass.): MIT-Press.

Johansen, Chr. 1862. Die nordfriesische Sprache nach der Föhringer und Amrumer Mundart. Kiel: Akademische Buchhandlung.

Laan, K. ter. 1953. Proeve van een Groninger spraakkunst. Winschoten: Van der Veen.
Lieber, R. 1983. 'Argument linking and compounds in English'. Linguistic Inquiry 14:251-285.

Pullum G.K. 1991. 'English nominal gerund phrases as noun phrases with verb-phrase heads'. Linguistics 29:763-799.

Roeper, Th. \& M.E.A. Siegel. 1978. 'A lexical transformation for verbal compounds'. Linguistic Inquiry 9:199-260.

Rosen, S. Th. 1989. 'Two types of noun incorporation: a lexical analysis'. Language 65:294-317.

Scalise, S. 1985. Generative Morphology. Dordrecht: Foris.

Schmidt-Petersen, J. \& J. Craigie. 1928. The North Frisian dialect of Föhr and Amrum. Edinburgh: I.B. Hutchen.

Schuurman, I. \& A. Wierenga. 1986. 'Syntactische nomenincorporatie bij infinitieven en deelwoorden'. In C. Hoppenbrouwers u.a. (eds.) Proeven van taalwetenschap. [= TABU 16 (1986)]. Groningen: Nederlands Instituut RUG, 339-350.

Shimamura, R. 1983. 'Backformation of English compound verbs'. In J.F. Richardson, M. Marks \& A. Chukerman (eds.). Papers from the parasession on the interplay of phonology, morphology and syntax. Chicago: Chicago Linguistic Society, 271-282.

Selkirk, E.O. 1982. The syntax of words. Cambridge (Mass.): MIT Press.

Sytstra, O.H. \& J.J. Hof. 1925. Nieuwe Friesche Spraakkunst. Leeuwarden: R. van der Velde.

Visser, W. 1989. 'Ta de nominale ynfinityf yn it Frysk'. Philologia Frisica anno 1988. Ljouwert: Fryske Akademy, 51-72.

Williams, E. 1981. 'On the notions "lexically related" and "head of a word"'. Linguistic Inquiry 12: 245-274.

Wilts, O. 1986. Wurdenbuk för Feer an Oomram. Norddorf, Amrum: Jens Quedens. 